\author{
ANNALES \\ POLONICI MATHEMATICI \\ XXVII (1972)
}

\title{
Absolute Nörlund summability factors of power series and Fourier series*
}

\author{
by Z. U. Ahmad (Aligarh, India)
}

1.1. Definitions. Let $\sum a_{n}$ be a given infinite series with the sequence of partial sums $\left\{s_{n}\right\}$. Let $\left\{p_{n}\right\}$ be a sequence of constants, real or complex, and let us write

$$
P_{n}=p_{0}+p_{1}+p_{2}+\ldots+p_{n} ; \quad P_{-1}=p_{-1}=0 .
$$

The sequence-to-sequence transformation

$$
t_{n}=\left(P_{n}\right)^{-1} \sum_{\nu=0}^{n} p_{n-\nu} s_{v} \quad\left(P_{n} \neq 0\right)
$$

defines the sequence $\left\{t_{n}\right\}$ of Nörlund means ([14], [19]) of the sequence $\left\{s_{n}\right\}$, generated by the sequence of coefficients $\left\{p_{n}\right\}$.

The series $\sum a_{n}$ is said to be absolutely summable $\left(N, p_{n}\right)$, or summable $\left|N, p_{n}\right|$, if

$$
\sum_{n}\left|t_{n}-t_{n-1}\right|<\infty, \quad[13]
$$

In the special case in which

$$
p_{n}=A_{n}^{a-1}=\frac{\Gamma(n+\alpha)}{\Gamma(\alpha) \Gamma(n+1)} \quad(\alpha>-1),
$$

the Nörlund mean reduces to the familiar $(C, a)$-mean, $([7], \S 5.13)$. The summability $\left|N, p_{n}\right|$, with $p_{n}$ defined by (1.1.2), is thus the same as summability $|C, a|,([6],[10])$. Similarly, in the case in which

$$
\left\{\begin{array}{l}
p_{n}=1 /(n+1) \quad(n=0,1,2, \ldots), \\
P_{n}=1+\frac{1}{2}+\ldots+\frac{1}{n+1} \sim \log n, \quad \text { as } n \rightarrow \infty,
\end{array}\right.
$$

* This work was executed under the Senior Research Fellowship Scheme of the Council of Scientific and Industrial Research, at the Department of Postgraduate Studies and Research in Mathematics, University of Jabalpur, Jabalpur, India. 
the Nörlund mean reduces to 'harmonic mean' [7], and the summability $\left|N, p_{n}\right|$ is then the same as 'absolute harmonic summability'. It is well known that $\left|N,(n+1)^{-1}\right| \subset|C, \alpha|$ for every positive $a,[12]$.

1.2. If

$$
\sum_{\nu=1}^{n}\left|s_{\nu}\right|=O(n)
$$

as $n \rightarrow \infty$, the series $\sum a_{n}$ is said to be strongly bounded by Cesàro means of order 1 , or bounded $[C, 1]$. If

$$
\sum_{v=1}^{n} \frac{\left|s_{v}\right|}{v}=O(\log v)
$$

as $n \rightarrow \infty$, the series $\sum a_{n}$ is said to be strongly bounded by 'logarithmic means' with index 1 , or bounded $[R, \log n, 1]$, [1.5].

Let $\sigma_{n}$ and $\tau_{n}$ denote the $n$th $(C, 1)$-means of the sequences $\left\{s_{n}\right\}$ and $\left\{n a_{n}\right\}$ respectively, viz.,

$$
\sigma_{n}=\frac{1}{n+1} \sum_{\nu=0}^{n} \mho_{n} ; \quad \tau_{n}-\frac{1}{n+1} \sum_{\nu=1}^{n} v a_{n} .
$$

Then, since by an identity of Kogbetliantz [10], $n^{\prime}\left(\sigma_{n}-\sigma_{n-1}\right)=\tau_{n}$, the uth total variation of the sequence $\left\{\sigma_{n}\right\}$ is given by

$$
\sum_{v=1}^{n}\left|\sigma_{v}-\sigma_{\nu-1}\right|==\sum_{v=1}^{n} \frac{\left|\tau_{v}\right|}{v} .
$$

1.3. Let $f(t)$ be a periodic function, with period $2 \pi$, integrable in the sense of Lebesgue over $(-\pi, \pi)$, and let

$$
\frac{1}{2} a_{0}+\sum_{n=1}^{\infty}\left(a_{n} \cos n t-\vdash b_{n} \sin n t\right) \equiv \frac{1}{2} a_{0}+\sum_{n=1}^{\infty} A_{n}(t)
$$

denote the Fourier series of the function $f(t)$.

1.4. For any sequence $\left\{f_{n}\right\}$, we write

$$
\Delta^{0} f_{n}=f_{n}, \quad \Delta f_{n}=\Delta^{1} f_{n}=f_{n}-f_{n+1},
$$

and

$$
\Delta^{\prime} f_{n}==\sum_{v=0}^{\infty} A_{\nu}^{-0 \cdot-1} f_{n+v},
$$

provided this series is convergent.

If $h$ and $k$ are positive integers, we have

$$
\Delta^{h} \Delta^{k} f_{n}=\Delta^{h+k} f_{n}
$$


A sequence $\left\{\lambda_{n}\right\}$ is said to be convex $\left([20]\right.$, p. 93) if $\Delta^{2} \lambda_{n} \geqslant 0$, for $n=0,1,2, \ldots$

If $\left\{\lambda_{n}\right\}$ is a convex sequence such that the series $\sum n^{-1} \lambda_{n}$ is convergent, then it is known that

(i) $\log n \lambda_{n}=o(1)$, as $n \rightarrow \infty$ ([5], Lemma IV);

(ii) $\sum n \Delta^{2} \lambda_{n}<\infty$ ([5], Lemma IV);

(iii) $\sum n(\log n) \Delta^{2} \lambda_{n}([16]$, IJemma $\tilde{5})$.

We write

for $v \geqslant 1, n \geqslant 1$.

$$
P_{n, \nu} \equiv P_{n} p_{u}{ }_{\nu}-P_{n-\nu} p_{n}
$$

2.1. Introduction. It was proved by McFadden ([12], Theorem 2.28), that, if (i) $p_{n}$ is non-negative and non-increasing, with $p_{0}>0$, and (ii) $p_{n+1} / p_{n}$ is non-decreasing, then $\left|N, p_{n}\right| \subset|C, 1|$.

We raise the question as to what type of sequences of factors $\left\{\varepsilon_{n}\right\}$ can be chosen so that the series $\sum \varepsilon_{n} a_{n}$ may be summable $\left|N, p_{n}\right|$, with $p_{n}$ more general than that characterized above, whenever the series $\sum a_{n}$ is not summable $|C, 1|$, but the total variation of the $(C, 1)$-mean of $\sum a_{n}$ is of a certain order $\mu_{n}$, say, where $\left\{\mu_{n}\right\}$ is a positive non-decreasing sequence.

As an answer to this question we establish, in Theorem 1, a resutl on the absolute Nörlund summability factors for general infinite series, which, in view of (1.4.4), includes the following results as special cases:

THEOREM A. If $\sum a_{n}$ is bounded $[R, \log n, 1]$ and $\lambda_{n}$ is a convex sequence such that $\sum n^{r-1} \lambda_{n}<\infty$, then

(i) $\sum a_{n} \lambda_{n}$ is summable $|C, 1|$, ([15], Theorem 1);

(ii) $\sum n^{-1}(\log n+1) \lambda_{n} a_{n}$ is summable $\left|N,(n+1)^{-1}\right|([11]$, Theorem 2).

THEOREM B. If $\sum a_{n}$ is summable $|C, 1|$, then

(i) $\sum n^{-1}(\log \overline{n-1-1}) \lambda_{n} a_{n}$ is summable $\left|N,(n+1)^{-1}\right|,[18]$;

(ii) $\sum n^{-1} P_{n} \lambda_{n} a_{n}$ is summable $\left|N, p_{n}\right|,[9]$.

Our main object in the present paper is to establish, with the help of our Theorem 1, some general theorems (Theorems 2, 3, 4 and 5) on the absolute Nörlund summability factors of power series and Fourier series. It is interesting to note that, in view of (1.4.4), our theorems contain, as special cases, the following known results in this line.

TiIforem C. If $f(z)=\sum c_{n} z^{n}$ is a power serics of the complex class $L$, such that

$$
\int_{0}^{t}\left|f\left(e^{i t}\right)\right| d \theta=O(|t|),
$$

as $t \rightarrow+0$, and $\left\{\lambda_{n}\right\}$ is a convex sequence such that $\sum n^{-1} \lambda_{n}<\infty$, then 
(i) $\sum \lambda_{n} c_{n}$ is summable $|C, 1|([17]$, Theorem I);

(ii) $\sum n^{-1}(\log \overline{n+1}) \lambda_{n} c_{n}$ is summable $\left|N,(n+1)^{-1}\right|([11]$, Theorem 1$)$.

THEOREM D. If $\left\{\lambda_{n}\right\}$ is a convex sequence such that $\sum n^{-1} \lambda_{n}<\infty$, then

(i) the series $\sum \lambda_{n} A_{n}(x)$ is summable $|C, 1|$ for almost all values of $x$, $[5]$

(ii) the series $\sum n^{-1}(\log \overline{n+1}) \lambda_{n} A_{n}(x)$ is summable $\left|N,(n+1)^{-1}\right|$ for almost all values of $x$ ([11], Theorem 3 ).

ThEorem E ([17], Theorem II). If $F(x)$ is even, $F^{\prime}(x) \in L^{2}(-\pi, \pi)$,

$$
\int_{0}^{t}|F(x)|^{2} d x=O(t),
$$

as $t \rightarrow+0$, and if $\left\{\lambda_{n}\right\}$ is a convex sequence such that $\sum n^{-1} \lambda_{n}<\infty$, then the sequence $\left\{A_{n}\right\}$ of Fourier coefficients of $F(x)$ has the propery that $\sum \lambda_{n} A_{n}$ is summable $|C, 1|$.

Theorem F ([17], Theorem III). If $F(x)$ is even, $F(x) \in L(-\pi, \pi)$,

$$
\int_{0}^{t}|F(x)| d x=O(t),
$$

as $t \rightarrow+0$, and if $\left\{\lambda_{n}\right\}$ is a convex sequence such that $\sum n^{-1} \lambda_{n}<\infty$, then the sequence $\left\{A_{n}\right\}$ of Fourier coefficients of $F(x)$ has the property that $\sum(\log n+1)^{-1 / 2} \lambda_{n} A_{n}$ is summable $|C, 1|$.

2.2. We establish the following theorems:

THEOREM 1. Let $p_{0}>0, p_{n} \geqslant 0(n=1,2, \ldots)$, and let $\left\{p_{n}\right\}$ be nonincreasing. If

$$
\sum_{\nu=1}^{n} \frac{\left|\tau_{p}\right|}{\nu}=O\left(\mu_{n}\right),
$$

where $\left\{\mu_{n}\right\}$ is a positive non-decreasing sequence, and if the sequence $\left\{\varepsilon_{n}\right\}$ is such that

(i) $\varepsilon_{n} \mu_{n}=O(1), n \Delta \mu_{n}=O\left(\mu_{n}\right)$,

(ii) $\sum n \mu_{n}\left|\Delta^{2} \varepsilon_{n}\right|<\infty$,

then the series $\sum(n+1)^{-1} P_{n} \varepsilon_{n} a_{n}$ is summable $\left|N, p_{n}\right|$.

TheOREM 2. Let $p_{n}$ be the same as in Theorem 1. If $\left\{\varepsilon_{n}\right\}$ is such that

(i) $\log n \varepsilon_{n}=O(1)$,

(ii) $\sum n \log n\left|\Delta^{2} \varepsilon_{n}\right|<\infty$,

then the series $\sum(n+1)^{-1} P_{n} \varepsilon_{n} A_{n}(x)$ is summable $\left|N, p_{n}\right|$ for almost all values of $x$. 
THEOREM 3. Let $p_{n}$ be the same as in Theorem 1. If $F(x)$ is even, $F(x)$ $\epsilon L^{2}(-\pi, \pi)$,

$$
\int_{0}^{t}|F(x)|^{2} d x=O(t),
$$

as $t \rightarrow+0$, and if $\left\{\varepsilon_{n}\right\}$ satisfies the same conditions as in Theorem 2, then the sequence $\left\{A_{n}\right\}$ of Fourier coefficients of $F(x)$ has the property that $\sum(n+1)^{-1} P_{n} \varepsilon_{n} A_{n}$ is summable $\left|N, p_{n}\right|$.

THEOREM 4. Let $p_{n}$ be the same as in Theorem 1. If $F(x)$ is even, $F(x) \in L(-\pi, \pi)$,

$$
\int_{0}^{t}|F(x)| d x=O(t),
$$

as $t \rightarrow+0$, and if $\left\{\varepsilon_{n}\right\}$ satisfies the same conditions as in Theorem 2 , then the sequence $\left\{A_{n}\right\}$ of Fourier coefficients of $F(x)$ has the property that $\sum(n+1)^{-1}(\log n)^{-1 / 2} P_{n} \varepsilon_{n} A_{n}$ is summable $\left|N, p_{n}\right|$.

THEOREM 5. If $f(z)=\sum c_{n} z^{n}$ is a power series of the complex class $L$, such that

$$
\int_{0}^{t}\left|f\left(e^{i \theta}\right)\right| d \theta=O(|t|),
$$

as $t \rightarrow+0$, and if $\left\{\varepsilon_{n}\right\}$ satisfies the same conditions as in Theorem 2 , then $\sum(n+1)^{-1} P_{n} \varepsilon_{n} c_{n}$ is summable $\left|N, p_{n}\right|$.

2.3. We use the following lemmas in the sequel.

LemMA 1 [1]. Let $p_{0}>0, p_{n} \geqslant 0(n=1,2, \ldots)$, and let $\left\{p_{n}\right\}$ be nonincreasing. Then, for $v \geqslant 1$,

$$
\sum_{n=p}^{\infty} \frac{p_{n}}{P_{n} \bar{P}_{n-1}} p_{n-\downarrow} \leqslant \frac{K}{v}\left({ }^{1}\right) ;
$$

$$
\sum_{n=\nu}^{\infty} \frac{p_{n}}{P_{n} P_{n-1}}\left(P_{n}-P_{n-v}\right) \leqslant K ;
$$

$$
\begin{gathered}
\sum_{n=v}^{\infty} \frac{\left(p_{n-\nu}-p_{n}\right)}{P_{n-1}} \leqslant K ; \\
\sum_{n=\nu}^{\infty} \frac{\left|\Delta_{\nu} p_{n-\nu}\right|}{P_{n-1}} \leqslant \frac{K}{P_{\nu}}+\frac{K}{\nu} .
\end{gathered}
$$

(1) Throughout, $K$ denotes an absolute constant, not necessarily the same at each occurrence. 
Lemma 2. If $p_{n}$ satisfies the same conditions as in Lemma 1 , then, for $\nu \geqslant 1$,

(a)

$$
\begin{aligned}
& \sum_{n=v}^{\infty} \frac{P_{n, v}}{P_{n}} \frac{P_{n \cdots 1}}{P_{n-1}}, \\
& \sum_{n=v}^{\infty} \frac{\left|\Delta_{v} P_{n, v}\right|}{P_{n} P_{n-1}} \leqslant \frac{K}{P_{v}} .
\end{aligned}
$$

Proof. Since

$$
\sum_{n=v}^{\infty} \frac{P_{n, \nu}}{P_{n} P_{n-1}}=\sum_{n=v}^{\infty} \frac{p_{n}}{P_{n} P_{n-1}-\ldots}\left(P_{n}-P_{n-,}\right)+\sum_{n=v}^{\infty} \frac{\left(p_{n-\nu}-p_{n}\right)}{P_{n-1}},
$$

(a) follows from Lemma 1 (b) and (c).

Again, since

we have

$$
\Lambda_{v} P_{n, v}=P_{n} \cdot \Lambda_{v} p_{n-v}-p_{n-v} p_{n},
$$

$$
\begin{aligned}
\sum_{n=v}^{\infty} \frac{\left|A_{v} P_{n, v}\right|}{P_{n} P_{n-1}} & \leqslant \sum_{n=v}^{\infty} \frac{p_{n}}{P_{n} P_{n-1}} p_{n-v}+\sum_{n=v}^{\infty} \frac{\left|A_{v} p_{n-v}\right|}{P_{n-1}} \\
& \leqslant \frac{K}{v}+\frac{K}{P_{v}} \quad \text { (by Lenmma 1(a) and (d)) } \\
& =K \cdot \frac{v+1}{v} \cdot \frac{1}{P_{v}} \cdot \frac{P_{v}}{v+1}+\frac{K}{P_{v}} \leqslant \frac{K}{P_{v}}
\end{aligned}
$$

by hypothesis, since $P_{v} /(v+1)$ is non-increasing.

This completes the proof of the lemma.

Lemma 3 ([4], Lemma 1 ; see also [2], [3]). If $\varepsilon_{n}=O(1)$, then

$$
\Delta^{a+\beta} \varepsilon_{n}=\sum_{\nu=n}^{\infty} A_{v-n}^{-\beta-1} \Delta^{a} \varepsilon_{n}
$$

for $a \geqslant 0, \beta \geqslant-1, \alpha+\beta>0$. If $\varepsilon_{n}=o(1)$, the last condition may be replaced by $\alpha+\beta \geqslant 0$.

LEMma 4. Let $\left\{\mu_{n}\right\}$ be a positive non-decreasing sequence such that $n \Delta \mu_{n}=O\left(\mu_{n}\right)$, as $n \rightarrow \infty$. If the sequence $\left\{\varepsilon_{n}\right\}$ is such that

(i) $\mu_{n} \varepsilon_{n}=O(1)$, as $n \rightarrow \infty$,

(ii) $\sum n_{n} \mu_{n}\left|\Delta^{2} \varepsilon_{n}\right|<\infty$

then

(a) $\sum \mu_{n}\left|\Delta \varepsilon_{n}\right|<\infty$,

and

(b) $n \mu_{n} \Delta \varepsilon_{n}=O(1)$, as $n \rightarrow \infty$. 
Proof. (a) Since, by hypothesis (i), $\varepsilon_{n}=O(1)$, applying Lemma 3, we have

$$
\Lambda \varepsilon_{n}=\sum_{\nu=n}^{\infty} \Lambda^{2} \varepsilon_{\nu}
$$

and hence,

$$
\begin{aligned}
\sum_{n=1}^{\infty} \mu_{n}\left|\Delta \varepsilon_{n}\right| & =\sum_{n=1}^{\infty} \mu_{n}\left|\sum_{v=n}^{\infty} A^{2} \varepsilon_{v}\right| \leqslant \sum_{n=1}^{\infty} \mu_{n} \sum_{r=n}^{\infty}\left|\Delta^{2} \varepsilon_{v}\right| \\
& =\sum_{v=1}^{\infty}\left|\Lambda^{2} \varepsilon_{v}\right| \sum_{n=1}^{\nu} \mu_{n} \leqslant \sum_{v=1}^{\infty} v \mu_{v}\left|\Lambda^{2} \varepsilon_{v}\right| \leqslant K<\infty
\end{aligned}
$$

by hypothesis.

(b) We see that

$$
\begin{aligned}
\sum_{n=1}^{\infty}\left|\Delta\left(n \mu_{n} \Delta \varepsilon_{n}\right)\right| & \leqslant \sum_{n=1}^{\infty} n \mu_{n}\left|A^{2} \varepsilon_{n}\right|+\left|-\sum_{n=1}^{\infty} \mu_{n}\right| \Delta \varepsilon_{n+1}\left|-+^{-} \sum_{n=1}^{\infty}(n+1)\right| A \mu_{n}|| \Delta \varepsilon_{n+1} \mid \\
& \leqslant K+K \sum_{n=1}^{\infty} \mu_{n} \mid\left\langle\left|\varepsilon_{n+1}\right| \leqslant K<\infty\right.
\end{aligned}
$$

by hypothesis and (a). This means that $\left\{n \mu_{n} \Delta \varepsilon_{n}\right\}$ is a sequence of bounded variation, which implies that it is bounded, that is,

$$
n \mu_{n} \Delta \varepsilon_{n}=O(1), \quad \text { as } n \rightarrow \infty \text {. }
$$

ILEMMA 5. If $\bar{\varepsilon}_{n}=(n+1)^{-1} P_{n} \varepsilon_{n}$, then under the hypotheses of Theorem 1 ,

(i) $\sum_{\nu=1}^{\infty} \frac{\left|\bar{\varepsilon}_{v}\right|}{P_{v}}\left|\tau_{v}\right| \leqslant K$,

(ii) $\sum_{\nu=1}^{\infty}\left|\Delta \varepsilon_{v}\right|\left|\tau_{v}\right| \leqslant K$.

Proof. (i) We have, as $m \rightarrow \infty$,

$$
\begin{aligned}
\sum_{v=1}^{m} \frac{\left|\bar{\varepsilon}_{v}\right|}{P_{v}}\left|\tau_{v}\right| & =O\left(\sum_{v=1}^{m}\left|\varepsilon_{v}\right| \frac{\left|\tau_{v}\right|}{y}\right) \\
& =O\left(\sum_{v=1}^{m} \mu_{v}\left|\Delta \varepsilon_{v}\right|\right)+O\left(\left|\varepsilon_{m}\right| \mu_{m}\right)=O(1),
\end{aligned}
$$

by hypotheses and Lemma 4 (a).

(ii) Since

$$
\Delta \bar{\varepsilon}_{n}=\frac{P_{n}}{n+1}-\Delta \varepsilon_{n}-\frac{p_{n+1}}{n+1} \varepsilon_{n+1}+\frac{P_{n+1}}{n+2} \frac{\varepsilon_{n+1}}{n+1},
$$

we have

$$
\left|\Delta \bar{\varepsilon}_{n}\right|=O\left(\left|\Delta \varepsilon_{n}\right| \frac{P_{n}}{n}\right)+O\left(\frac{\left|\varepsilon_{n+1}\right|}{n}\right) .
$$


Hence, as $m \rightarrow \infty$,

$$
\begin{aligned}
\sum_{\nu=1}^{m}\left|\Delta \bar{\varepsilon}_{v}\right|\left|\tau_{v}\right| & =O\left(\sum_{\nu=1}^{m} P_{v}\left|\Lambda \varepsilon_{v}\right| \frac{\left|\tau_{v}\right|}{\nu}\right)+O\left(\sum_{v=1}^{m}\left|\varepsilon_{v+1}\right| \frac{\left|\tau_{v}\right|}{\nu}\right) \\
= & O\left(\sum_{\nu=1}^{m-1} v \mu_{v}\left|\Lambda^{2} \varepsilon_{v}\right|\right)+O\left(\sum_{\nu=1}^{m-1} \mu_{v}\left|\Lambda \varepsilon_{v+1}\right|\right)+ \\
& +O\left(m \mu_{m}\left|\Lambda \varepsilon_{m}\right|\right)+O\left(\mu_{m+1}\left|\varepsilon_{m+1}\right|\right) \\
= & O(1),
\end{aligned}
$$

by hypotheses and Lemma 4 (a) and (b).

This completes the proof of the lemma.

LEMмa 6 [17]. If $f(z)=\sum c_{n} z^{n}$ is a power series of complex class $L$, such that

$$
\int_{0}^{t}\left|f\left(e^{i \theta}\right)\right| d \theta=O(|t|),
$$

as $t \rightarrow+0$, then $\sum c_{n}$ is bounded $[R, \log n, 1]\left({ }^{2}\right)$.

JEMMA 7 ([15], p. 294). If $\sum a_{n}$ is bommded $[R, \log n, 1]$, then

$$
\sum_{v=1}^{n} \frac{\left|\tau_{v}\right|}{y}=O(\log n)
$$

as $n \rightarrow \infty$.

Lemma 8. If $\sum a_{n}$ is bounded $[C, 1]$, then it is bounded $[R, \log n, 1]$.

The proof is easy.

LAgMma 9. ([5], I Jemma 2). Let

$$
\tau_{n}(x)=\frac{1}{n+1} \sum_{\nu=1}^{n} \nu A_{v}(x) .
$$

Then

$$
\sum_{\nu=1}^{n}\left|\tau_{\nu}(x)\right|=o(n)
$$

for almost all values of $x$.

LEMMA 10. If $\tau_{n}(x)$ is defined as in Lemma 9, then

$$
\sum_{\nu=1}^{n} \frac{\left|\tau_{\nu}(x)\right|}{\nu}=o(\log n),
$$

as $n \rightarrow \infty$, for almost all values of $x$.

(2) This is the ' $O$ ' version of a previous result of Hardy and Littlewood [8]. 
Proof. By Abel's transformation and Lemma 9, we have

$$
\begin{aligned}
\sum_{\nu=1}^{n} \frac{\left|\tau_{\nu}(x)\right|}{\nu} & =\sum_{\nu=1}^{n} \frac{1}{\nu(\nu+1)} \sum_{\mu=1}^{v}\left|\tau_{\mu}(x)\right|+\frac{1}{n+1} \sum_{\mu=1}^{n}\left|\tau_{\mu}(x)\right| \\
& =o(\log n)+o(1)=o(\log n)
\end{aligned}
$$

as $n \rightarrow \infty$, for almost all values of $x$.

Lemma 11 ([17], Lemma 4). Let $F(x)$ be even, $F(x) \in L^{2}(-\pi, \pi)$, and let $S_{n}$ denote the nth partial sum of its Fourier series at the origin. Then, if

$$
\int_{0}^{\theta}|F(x)|^{2} d x=O(\theta)
$$

as $\theta \rightarrow+0,\left\{\mathbb{S}_{n}\right\}$ will be summble $[C, 1]$.

Lемма 12 [17]. Let $F(x)$ be even, $F(x) \in L(-\pi, \pi)$, and let $S_{n}$ denote the nth partial sum of its Fourier series at the origin. Then, if

$$
\int_{0}^{\theta}|F(x)| d x=O(0)
$$

as $\theta \rightarrow+0$, then

$$
\sum_{v=1}^{n}\left|S_{v}\right|=O\left\{n(\log n)^{1 / 2}\right\} .
$$

2.4. Proof of Theorem 1. Let $\bar{\varepsilon}_{n}=(n+1)^{-1} P_{n} \varepsilon_{n}$, and let $t_{n}^{*}$ denote the $n$th Nörlund mean of the series $\sum \bar{\varepsilon}_{v} a_{v}$. Then, by definition, we have

$$
t_{n}^{*}=\frac{1}{P_{n}} \sum_{v=0}^{n} p_{n-\nu} \sum_{\mu=0}^{v} \bar{\varepsilon}_{\mu} a_{\mu}=\frac{1}{P_{n}} \sum_{v=0}^{n} P_{n-,} \bar{\varepsilon}_{v} a_{v}
$$

and

$$
\begin{aligned}
t_{n}^{*}-t_{n-1}^{*}= & \frac{1}{P_{n} P_{n-1}} \sum_{v=1}^{n}\left(P_{n} p_{n-v}-P_{n-v} p_{n}\right) \bar{\varepsilon}_{v} a_{v} \\
= & \frac{1}{P_{n} P_{n-1}} \sum_{v=1}^{n} P_{n, v} \bar{\varepsilon}_{v} a_{v} \\
= & \frac{1}{P_{n} P_{n-1}} \sum_{v=1}^{n} P_{n, v} \frac{\bar{\varepsilon}_{v}}{v} \tau_{v}+\frac{1}{P_{n} P_{n-1}} \sum_{v=1}^{n} P_{n, v} \Delta \bar{\varepsilon}_{v} \tau_{v}+ \\
& +\frac{1}{P_{n} P_{n-1}}-\sum_{\nu=1}^{n} \Delta_{v} P_{n, v} \bar{\varepsilon}_{v+1} \tau_{v}=\frac{1}{P_{n} P_{n-1}}\left(\Sigma_{1}+\Sigma_{2}+\Sigma_{3}\right)
\end{aligned}
$$

say.

2 - Annales Polonici Mathematici xxvI 
Therefore, in order that $\sum_{n}\left|t_{n}^{*}-t_{n-1}^{*}\right| \leqslant K$, it is sufficient to show that

$$
\sum_{n} \frac{1}{P_{n} P_{n-1}}\left|\Sigma_{r}\right| \leqslant K \quad(r=1,2,3) .
$$

Now, first, we have

$$
\begin{aligned}
\sum_{n=1}^{\infty} \frac{1}{P_{n} P_{n-1}}\left|\Sigma_{1}\right| & \leqslant \sum_{n=1}^{\infty} \frac{1}{P_{n} P_{n-1}} \sum_{\nu=1}^{n} P_{n, v}\left|\bar{\varepsilon}_{v}\right| \frac{\left|\tau_{v}\right|}{\nu} \\
& =\sum_{v=1}^{\infty}\left|\bar{\varepsilon}_{v}\right| \frac{\left|\tau_{\nu}\right|}{\nu} \sum_{n=v}^{\infty} \frac{P_{n, \nu}}{P_{n} P_{n-1}} \\
& \leqslant K \sum_{\nu=1}^{\infty}\left|\bar{\varepsilon}_{v}\right| \frac{\left|\tau_{v}\right|}{v} \quad \text { (by Lemma 2(a)) } \\
& \leqslant K \sum_{v=1}^{\infty} \frac{\left|\bar{\varepsilon}_{v}\right|}{P_{\nu}}\left|\tau_{v}\right| \leqslant K
\end{aligned}
$$

by hypotheses and Lemma 5 (i).

Next, we have

$$
\begin{aligned}
\sum_{n=1}^{\infty} \frac{1}{P_{n} P_{n-1}}\left|\Sigma_{2}\right| & \leqslant \sum_{n=1}^{\infty} \frac{1}{P_{n} P_{n-1}} \sum_{v=1}^{n} P_{n, v}\left|\Delta \bar{\varepsilon}_{v}\right|\left|\tau_{v}\right| \\
& =\sum_{v=1}^{\infty}\left|\Delta \bar{\varepsilon}_{v}\right|\left|\tau_{v}\right| \sum_{n=v}^{\infty} \frac{P_{n, v}}{P_{n} P_{n-1}} \\
& \leqslant K \sum_{v=1}^{\infty}\left|\Delta \bar{\varepsilon}_{v}\right|\left|\tau_{v}\right| \quad \text { (by Lenma 2(a)) } \\
& \leqslant K
\end{aligned}
$$

by hypotheses and Lemma 5 (ii).

Finally, we have

$$
\begin{aligned}
\sum_{n=1}^{\infty} \frac{1}{P_{n} P_{n-1}}\left|\Sigma_{3}\right| & \leqslant \sum_{n=1}^{\infty} \frac{1}{P_{n} P_{n-1}} \sum_{v=1}^{n}\left|\Delta_{v} P_{n, v}\right|\left|\bar{\varepsilon}_{v+1}\right|\left|\tau_{v}\right| \\
& \left.\leqslant K \sum_{v=1}^{\infty} \frac{\left|\vec{\varepsilon}_{v+1}\right|}{P_{v}}\left|\tau_{v}\right| \quad \text { (by Isemma } 2(\mathrm{~b})\right) \\
& \leqslant K,
\end{aligned}
$$

by hypotheses and Iemma $5(\mathrm{i})$.

This terminates the proof of Theorem 1 . 
2.5. Proof of Theorems 2,3,4 and 5. We obtain Theorem 2 from Theorem 1 , with $\mu_{n}=\log n$, by an appeal to Lemma 10 .

Theorem 3 can easily be obtained from Theorem 1, with $\mu_{n}=\log n$, by successive applications of Lemmas 11,8 and 7 .

We get Theorem 4 from Theorem 1 with $\mu_{n}=(\log n)^{3 / 2}$, and with $\varepsilon_{n} /(\log n)^{1 / 2}$ in place of $\varepsilon_{n}$, by an appeal to Lemma 12 and by using the fact that

implies

$$
\sum_{\nu=1}^{n}\left|S_{\nu}\right|=O\left\{n(\log n)^{1 / 2}\right\}
$$

$$
\sum_{v=1}^{n} \frac{\left|\tau_{v}\right|}{\nu}=O\left\{(\log n)^{3 / 2}\right\}
$$

Finally, we obtain Theorem 5 from Theorem 1 with $\mu_{n}=\log n$, by appealing to Lemmas 6 and 7 .

I take this opportunity to express my sincere thanks to Professor T. Pati for his kind encouragement and to the Council of Scientific and Industrial Research for their financial support during the preparation of this paper.

\section{References}

[1] Z, U. Ahmad, On the absolute Nörlund summability factors of a Fourier series, Riv. Mat. Univ. Parma (2), 7 (1966), p. 157-169.

[2] A. F. Andersen, Studier over Cesàro summabilitets-metode, Copenhagen 1921.

[3] - Comparison theorems in the theory of Cesàro summability, Proc. London Math. Soc. (2), 27 (1928), p. 39 - 71.

[4] L. S. Bosanquet, Note on Bohr-Hardy theorem, J. London Math. Soc. 17 (1942), p. $166 \cdot 173$.

[5] H. C. Chow, On the summability factors of Fourier series, ibidem 16 (1941), p. $215 \cdot 220$.

[6] M. Fekete, Zur Theorie der divergenten Reihen, Math. és Termes, Ersitö (Budapest), 29 (1911), p. $719-726$.

[7] G. H. Hardy, Divergent series, Oxford 1949.

[8] - and J. E. Littlewood, The strong summability of Fourier series, Fund. Math. 25 (1935), p. 162 - 189.

[9] Nand Kishore, On the absolute Norlund summability factors, Riv. Mat. Univ. Parma (2), 6 (1965), p. 129 - 134.

[10] E. Kogbetliantz, Sur les séries absolument sommable par la méthode des moyennes arithmétiques, Bull. Sci. Math. (2), 49 (1925), p. 234 - 256.

[11] S. N. Lal, On the absolute harmonic summability of the factored power series on its circle of convergence, Indian J. Math. 5 (1963), p. 55 - 66.

[12] L. McFadden, Absolute Nörlund summability, Duke Math. J. 9 (1942), p. $168 \cdot 207$.

[13]. F. M. Mears, Absolute regularity and the Norlund mean, Ann. of Math. 38 (1937), p. $594 \cdot 601$. 
[14] N. E. Nörlund, Sur une application des fonction permutables, Lunds Universitets Årsskrift (2), 16 (1919), No. 3.

[15] T. Pati, Absolute Cesàro summability factors of infinite series, Math. Zeitschr. 78 (1962), p. $293-297$.

[16] - and S. R. Sinha, On the absolute summability factors of Fourier series, Indian J. Math. 1 (1958), p. 41 - 54.

[17] C. T. Rajagopal, On $|C, 1|$ summability factors of power series and Fourier series, Math. Zeitschr. 80 (1963), p. 265 - 268.

[18] T. Sing h, The absolute harmonic summability factors of infinite series, Abstracts, Proc. combined 5lst and 52nd sessions, Indian Science Congress Assoc., 1965.

[19] G. F. Woronoi, Extension of the notion of the limit of the sum of terms of an infinite series, Ann. of Math. 33 (1932), p. 422 - 428.

[20] A. Zygmund, Trigonometric series, Vol. I, Cambridge 1959.

DEPARTMENT OF MATHEMATICS AND STATISTICS

ALIGARH MUSLIM UNIVERSITY

ALIGARH, INDIA

Reçu par la Rédaction le 2. 3. 1970 